\title{
Development of psychotic symptoms following ingestion of small quantities of alcohol
}

\author{
This article was published in the following Dove Press journal: \\ Neuropsychiatric Disease and Treatment \\ 22 September 2016 \\ Number of times this article has been viewed
}

\author{
Da-Li Lu' \\ Xiao-Ling Lin ${ }^{2}$ \\ 'Department of Psychiatry, Xiamen \\ Xianyue Hospital, Xiamen, People's \\ Republic of China; ${ }^{2}$ School of Nursing, \\ Sun Yat-Sen University, Guangzhou, \\ People's Republic of China
}

\begin{abstract}
Psychotic symptoms can occur in some clinical conditions related to alcohol, such as intoxication, withdrawal, and other alcohol-induced neuropsychiatric disorders. Here, we present a case report of a 24-year-old man, without a known psychiatric history, who developed brief psychotic symptoms following ingestion of small quantities of alcohol repeatedly. To our knowledge, no related previous literature regarding this has been reported.
\end{abstract}

Keywords: Alcohol consumption, psychotic symptoms, behavioral change, minimal quantity, pathological intoxication, schizophrenia

\section{Introduction}

Patients with alcohol abuse and dependence may suffer from psychiatric disorder, and there is a high prevalence of alcohol and other drug use disorders among patients with schizophrenia and other psychotic disorders - shown to be as high as $50 \%$ in some studies. ${ }^{1-3}$ A high prevalence of alcohol abuse and substance use disorders has also been found in patients with first-episode psychosis. ${ }^{4,5}$ While alcohol-induced psychotic disorder is a well-recognized clinical disorder, relatively little is known about the mechanism of this condition. Previous studies have reported decreased $\gamma$-aminobutyric acid (GABA, inhibitory) ${ }^{6}$ and increased plasma glutamate and aspartate in patients who consume alcohol, ${ }^{7}$ while a placebo-controlled study reported superior efficacy of the inhibitory neurotransmitter glycine in reducing hallucinations in patients. ${ }^{7}$ Certain genetic variants (alleles), particularly the ADH1B*2, ADH1B*3, ADN1C*1, and ALDH2*2 alleles, which code for alcohol-metabolizing enzymes such as alcohol dehydrogenase (ADH) and aldehyde dehydrogenase (ALDH), have been associated with lower rates of alcohol dependence. ${ }^{8}$ The presence of these alleles may lead to an accumulation of acetaldehyde during the alcohol metabolism process, which can result in heightened subjective and objective effects. ${ }^{8}$

Furthermore, the existence of an acute brain syndrome manifested by psychotic reaction to alcohol without regard to the amount of alcohol consumed has been welldescribed in the past. ${ }^{9,10}$ The acute, chaotic disruption of behavior resulting from ingestion of a small quantity of alcohol, known as "pathological intoxication", has long been recognized as a psychiatric entity. ${ }^{11}$ Pathological intoxication (PI), also called alcohol idiosyncratic intoxication, was said to occur predominantly in persons with low tolerance to alcohol, but its existence as a definable syndrome is still controversial. ${ }^{12}$ The disease has been defined as an acute brain syndrome manifested by a marked behavioral or psychotic reaction after minimal alcohol intake in people with no preexisting mental disorder. ${ }^{12}$ The essential points of all the definitions of this disease were the following: 1) marked maladaptive behavioral change (usually aggressive or assaultive behavior) 
with minimal alcohol intake, 2) the behavior is atypical of the person when not drinking, and 3) cause not being any other physical or mental disorder according to DSM-III-R (Diagnostic and Statistical Manual of Mental Disorders Third Edition Revised). ${ }^{13}$ Although PI had been listed in previous DSM editions, DSM-IV omitted it because of lack of supporting evidence to show that it was distinct from regular alcohol intoxication. Furthermore, such a condition of PI would be mostly likely diagnosed as alcohol intoxication or alcoholrelated disorder according to DSM-IV to DSM-V.

There was also some evidence of PI in the People's Republic of China, and only ten published case reports of PI have been identified upon a Chinese language literature review from 1984 to 2012 . The data for this review were based on the Chinese language literature identified from searches of the China National Knowledge Infrastructure (www.cnki.net; 1979-2012), which is the largest online Chinese language literature database. The clinical specifics related to these published cases are summarized in Table 1. Blood alcohol concentration was not measured in any patient, none of them showed unsteady gait and were inarticulate. Besides, all had absolute aggressive behavior changes, and the duration of the episode was quite short and ceased after a few minutes to several hours. Most of them were young, and four of them had accompanying schizophrenic symptoms such as visual hallucination or delusion.

In the current study, we report a 24-year-old man, without a known psychiatric history of psychotic or affective disorder, who developed a unique, brief psychotic disorder following ingestion of small quantities of alcohol, which seems very different from PI.

\section{Case presentation}

The patient provided written informed consent for publication of this case report. Mr Z. is a 24-year-old, previously healthy college student who developed psychotic features after drinking beer on the evening of June 15, 2011. Ten minutes after having drunk two glasses $(\sim 200 \mathrm{~mL})$ of beer (alcoholic strength of the beer was less than 4\%), he was agitated and screaming, saying that his parents were in danger and that he should go to save them. He also masturbated in public without feeling shy although there were two female classmates around. Following this, he was sent to the hospital immediately. At the time of admission, he presented with unusual thought processes and disorganized thinking. At the hospital, he demanded strongly that the door be closed and curtains be drawn over the windows. When the nurses switched on the light, he would scream at them. When he saw his father, he grabbed both of his father's hands tightly as if he feared losing his father. He closed his eyes but did not sleep the entire night, and he also called his father's name every 5 minutes to ensure that his father was around. If his father did not respond, he would feel nervous. He looked at the curtain all through the night from the bed, watching the shadow that the light of street lamp cast through the curtain, and listened to the noise of cars as if waiting for something to happen. He felt that the living environment around him was not as usual.

Nuclear magnetic resonance imaging (MRI) scan of the brain and 24-hour ambulatory electroencephalogram examination revealed no remarkable changes. Self-Rating Anxiety Scale and Self-Rating Depression Scale were administered to him, and the scores of the two scales were 50 and 28. There were no other mental records for him as he was unwilling to complete any more tests. At the time of admission, blood was routinely drawn to determine the blood alcohol level, perform routine blood examination, and assess hepatic and renal function and serum electrolyte level. Blood alcohol level on admission was $30 \mathrm{mg} / \mathrm{dL}$ (6.52 mmol/L), and other blood examination results were normal. Besides, the time span between ingestion of alcohol and blood alcohol level testing was very short - it was approximately 2 or 3 hours. There was no evidence of auditory or visual hallucination, delusions of reference, or other psychotic symptoms. He had not experienced stress or other traumatic experiences before. Besides, there was a drinking history for the patient: he had consumed alcohol only one or two times in the past 3 months - far from alcohol abuse and dependence.

For this reason, it was considered that the current situation was an episode of brief psychotic symptoms, but not schizophrenia spectrum or other psychotic disorders. So, the doctor began therapy with Seroquel (Quetiapine) $25 \mathrm{mg}$ Qn initially. Four days later, the dose was increased to $50 \mathrm{mg} /$ night. The patient began showing great improvement within 7 days after admission. The doctor felt that his mental state returned to normal and that his symptoms resolved completely. He was discharged on the treatment of Seroquel (Quetiapine) $50 \mathrm{mg} /$ night. When he went back school 1 week later, he did not take medication as before. He still studied well in school as before and showed no unusual behavior or symptoms.

Approximately 40 days later, he had drunk approximately $150 \mathrm{~mL}$ of beer with his father and his relatives. Again, the symptoms recurred, similar to the first time. His father sent him to the hospital immediately. When we saw him, he was too nervous to talk with us. There was evidence of thought blocking. We started therapy with Seroquel (Quetiapine) 


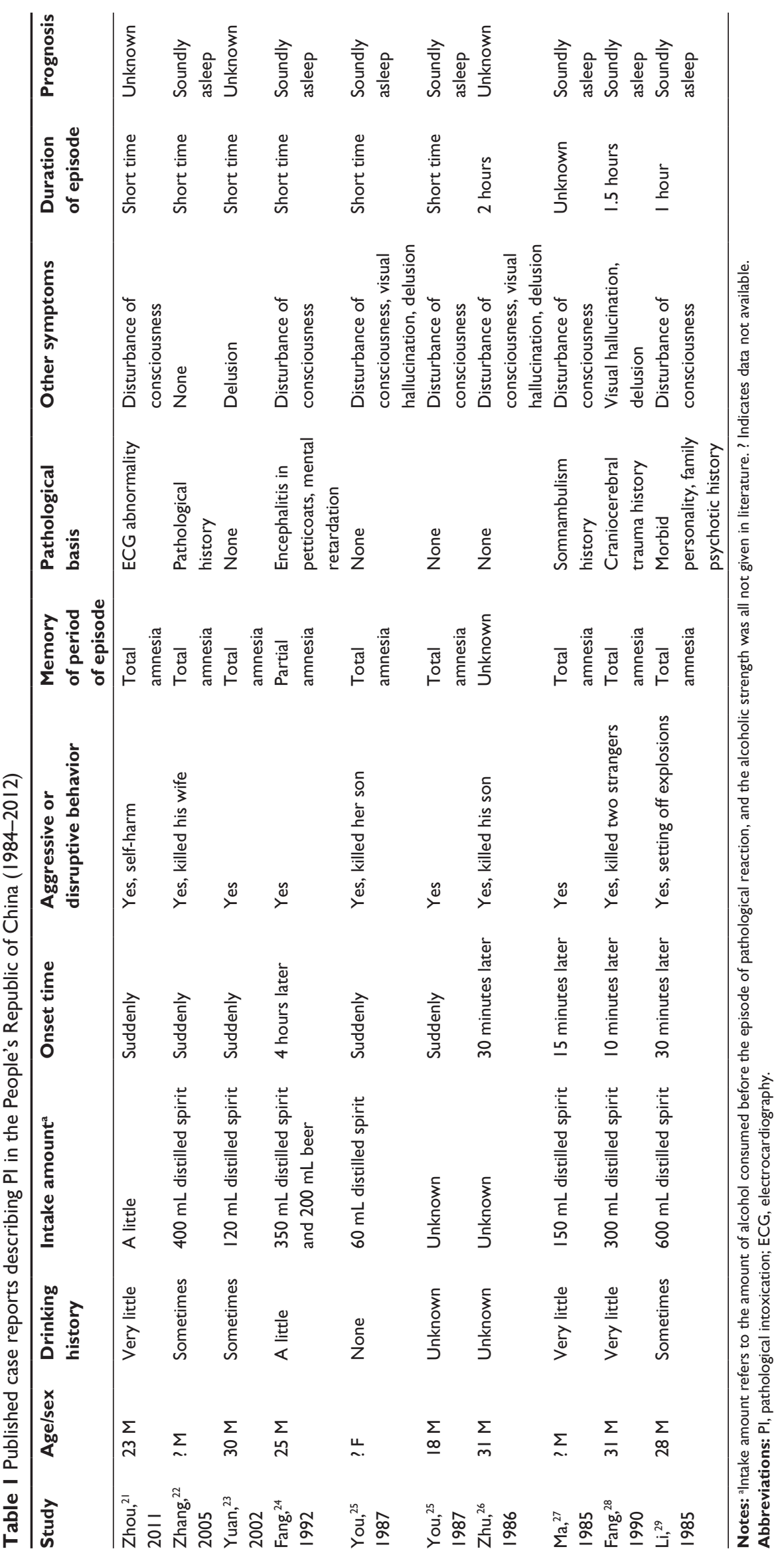


$25 \mathrm{mg} /$ night (as before). Furthermore, the patient did not experience any psychotic symptoms or behavior change in between these two episodes. Three days later, he returned to normal. He partly remembered what he had done 3 days before. As his symptoms resolved completely, his father stopped him from continuing the drug. Approximately 30 days later, his symptoms recurred again because he had drunk three glass of beer, approximately $150 \mathrm{~mL}$. He was suggested to continue taking Seroquel (Quetiapine) $25 \mathrm{mg} / \mathrm{night}$. His mental state returned to normal and his symptoms resolved completely 4 days after he took the medicine. We continued to contact the patient and his family for the following 3 months. He has since stopped taking the medicine and also does not drink alcohol any more. He also continued performing well at university (just as before).

\section{Discussion}

In the present report, we described the case of a young patient who presented with three episodes of behavioral change of acute onset suggestive of the brief psychotic episodes. Interestingly, each of the hospital admissions was triggered by small quantities of alcohol consumption. The symptoms manifested mainly as thought blocking; psychosensory disturbance; public masturbation; insecurity without elevated, expansive, irritable mood; or other psychotic symptoms. The symptoms occurred and ended abruptly, at around 3-7 days. The patient returned back to normal after therapy. Both the MRI scanning of the patient's brain and 24-hour ambulatory electroencephalogram were unremarkable. Furthermore, the patient and his family had no history of psychotic or affective disorder, personality disorder, or a seizure disorder. There was a very light history of drinking for the patient, and there was no weird behavior before when he consumed alcohol. What surprised us most was that once he had drunk such a small amount alcohol, his behavioral reaction started to emerge. To our knowledge, there is no related literature reporting the occurrence of such type of symptoms after ingestion of a small quantity of alcohol.

Alcohol addiction or withdrawal could induce psychotic symptoms, ${ }^{14}$ but it seems that the patient in our case was far from such circumstances. The patient had no history of alcohol abuse and dependence, but had drunk beer only one or two times in the 3 months before the first episode. Also, blood alcohol concentration at admission was low (only $6.52 \mathrm{mmol} / \mathrm{L}$ ), and the amount of beer ingested was small and the alcoholic strength of beer he consumed was less than $4 \%$. Several differences should be pointed out when comparing these disease characteristics to that of PI. In our case, the patient experienced a marked behavioral change, such as public masturbation, but no aggressive or assaultive behavior. In addition, the duration of the episode in our patient ( $\sim 3-7$ days) was much longer than that in the cases with PI, which lasts for only several minutes or hours. ${ }^{10-12}$ Besides, most PI patients presented total amnesia for the period of the episode, ${ }^{12}$ while in our case the patient had partial amnesia. Subsequently, when we used Seroquel to treat the patient, he started to do better. Also, episode of PI normally end with sound asleep. ${ }^{10-12}$ In conclusion, there was insufficient evidence to demonstrate that the patient might suffer from PI or other alcohol-related neuropsychiatric disorders, although the episodes were induced by ingestion of a tiny amount of alcohol.

It seems still more difficult for us to ascertain the etiology of the presentation. Although the patient showed atypical psychotic symptom without evident hallucination or delusion in these three episodes, the childish mannerisms and bizarre behavior still relates to or is characteristic of schizophrenia. Besides, the age of onset is 24 -year-old (very young). However, the one noteworthy point was that the patient would return to normal every time after therapy with Seroquel (Quetiapine). As the evidence accumulates, we have more confidence to speculate that the patient might be exhibiting early symptoms of schizophrenia and that ingestion of small amount of beer might induce the episode accidentally. In other words, ingestion of small amount of alcohol might be one of the predisposing factors of the episode of atypical psychotic symptoms in our case. Theoretically speaking, other issues, such as negative life events, might also induce psychosis.

The patient presented with irritability, psychomotor agitation, and insomnia after drinking beer, which also fulfils the DSM-IV criteria for catatonia. Catatonia is a clinical syndrome characterized by alterations in motor behavior, and changes in thought and mood and can occur in the context of several disorders, including neurodevelopmental, psychotic, bipolar, depressive disorders, and other medical conditions. Catatonia has been documented as occurring in alcohol withdrawal in rodents, and rarely in humans, but not occurring in alcohol drinking. ${ }^{15,16}$ Another possible diagnosis for this case was delirium. A patient with general delirious symptoms including thought process and sensory disturbances may fulfill the DSM-IV criteria for delirium. However, our patient exhibited no clouded consciousness, disorientation, or disturbed circadian rhythms, so this was not a possible diagnosis in this case. 
Another diagnosis of this case considered was anxiety disorder associated with substance intoxication. Clinical studies have documented a significant degree of comorbidity between anxiety disorders and alcohol use disorders. Previous studies, including both animal and human, have shown that acute exposure to low-to-moderate doses of ethanol are anxiolytic, ${ }^{17,18}$ and ingestion of small amount of ethanol resulted in development of anxiety symptoms in our patient. The etiological nature of this relationship is not well understood. One possible reason for this could be that acute ethanol ingestion in our patient was associated with an acute decrease in GABA concentrations of the brain, and studies (in humans and animals) have shown that GABA deficits may induce stress and anxiety. ${ }^{19,20}$

Taken together, this case demonstrates a special presentation of a marked behavioral change, which is suggestive of an episode of brief psychotic symptoms, after ingestion of small quantities of alcohol. And we can only assume, from the evidence and analysis, what disease the patient might be having. As a psychiatrist, it is essential to advise the patient not to drink any more, and, satisfactorily, he was also studying well at school during the follow-up period of 3 months. Further close follow-up with the patient and his family is required.

\section{Acknowledgment}

The study was supported by grants from The Young Teachers Program of Sun Yat-Sen University (53000-31121400).

\section{Disclosure}

The authors report no conflicts of interest in this work.

\section{References}

1. Addington J, Addington D. Patterns, predictors and impact of substance use in early psychosis: a longitudinal study. Acta Psychiatr Scand. 2007; 115:304-309.

2. Petersen L, Jeppesen P, Thorup A, et al. Substance abuse and first-episode schizophrenia-spectrum disorders. The Danish OPUS trial. Early Interv Psychiatry. 2007;1(1):88-96.

3. Swartz MS, Wagner HR, Swanson JW, et al. Substance use and psychosocial functioning in schizophrenia among new enrollees in the NIMH CATIE study. Psychiatr Serv. 2006;57(8):1110-1116.

4. Regier DA, Farmer ME, Rae DS, et al. Comorbidity of mental disorders with alcohol and other drug abuse. Results from the Epidemiologic Catchment Area (ECA) Study. JAMA. 1990;264(19):2511-2518.

5. Margolese HC, Malchy L, Negrete JC, Tempier R, Gill K. Drug and alcohol use among patients with schizophrenia and related psychoses: levels and consequences. Schizophr Res. 2004;67(2-3):157-166.

6. Jordaan GP, Emsley R. Alcohol-induced psychotic disorder: a review. Metab Brain Dis. 2014;29(2):231-243.
7. Aliyev NA, Aliyev ZN. Application of glycine in acute alcohol hallucinosis. Hum Psychopharmacol. 2005;20(8):591-594.

8. Wall TL, Luczak SE, Hiller-Sturmhöfel S. Biology, Genetics, and Environment: underlying factors influencing alcohol metabolism. Alcohol Res. 2016;38(1):59-68.

9. Banay RS. Pathologic reaction to alcohol. I. Review of the Literature and original case report. Q J Stud Alcohol. 1944;4:580-605.

10. Maletzky BM. The diagnosis of pathological intoxication. J Stud Alcohol. 1976;37(9):1215-1228.

11. Bach-y-Rita G, Lion JR, Ervin FR. Pathological intoxication: clinical and electroencephalographic studies. Am J Psychiatry. 1970;127(5): 698-703.

12. Perr IN. Pathological intoxication and alcohol idiosyncratic intoxicationPart I: Diagnostic and clinical aspects. J Forensic Sci. 1986;31(3): 806-811.

13. American Psychiatric Association. Diagnostic and Statistical Manual of Mental Disorders: DSM-III-R. 3rd edition revised. Washington, DC: American Psychiatric Association; 1987:129.

14. Perälä J, Kuoppasalmi K, Pirkola S, et al. Alcohol-induced psychotic disorder and delirium in the general population. Br J Psychiatry. 2010; 197(3):200-206.

15. Uzbay IT. L-NAME precipitates catatonia during ethanol withdrawal in rats. Behav Brain Res. 2001;119(1):71-76.

16. Muralidharan K, Rajkumar RP, Ananthapadmanabha Rao S, Benegal V. Catatonia as a presenting feature of alcohol withdrawal: a case report. Prim Care Companion J Clin Psychiatry. 2007;9(6):465.

17. Koob GF. A role for GABA mechanisms in the motivational effects of alcohol. Biochem Pharmacol. 2004;68(8):1515-1525.

18. Kushner MG, Abrams K, Borchardt C. The relationship between anxiety disorders and alcohol use disorders: a review of major perspectives and findings. Clin Psychology Rev. 2000;20(2):149-171.

19. Johnson PL, Truitt WA, Fitz SD, Lowry CA, Shekhar A. Neural pathways underlying lactate-induced panic. Neuropsychopharmacology. 2008;33(9):2093-2107.

20. Goddard AW, Mason GF, Almai A, et al. Reductions in occipital cortex GABA levels in panic disorder detected with $1 \mathrm{~h}$-magnetic resonance spectroscopy. Arch Gen Psychiatry. 2001;58(6):556-561.

21. Zhou YJ, Liu XX. Pathological drunkenness: a case report. Journal of Clinical Psychosomatic Diseases. 2011;17(3):196. Chinese.

22. Zhang DW. How to deal with the behavior of "causing the death" by pathological drunkenness person. Procuratorate Daily. 2005, March, 25. Chinese.

23. Yuan SX, Guan J, Li F. Criminal responsibility ability analysis under different types of drunkenness states. Professional committee of the Chinese society of forensic judicial psychiatry 1 st national conference on forensic psychiatry. June 2002; Chengdu, People's Republic of China. Chinese.

24. Fang MZ, Cui YH, Li CP. The clinical presentation and diagnosis of abnormal alcoholic intoxication in forensic psychiatric expertise. Chinese Mental Health Journal. 1992;6(2):69-71. Chinese.

25. You W. The further study for the criminal responsibility of the person with pathological drunkenness. Shangdong Law. 1987;2:29-31. Chinese.

26. Zhu BS. Analysis of criminal responsibility of person with pathological drunkenness. Legal study and research. 1986;7:68-69. Chinese.

27. Ma ZY. The relationship between alcoholic intoxication and law. Journal of Hebei University. 1985;4:162-163. Chinese.

28. Fang MZ. Clinical characteristics and criminal responsibility of pathological drunkenness. Medium Medical Journal. 1990;25(3):27-29. Chinese.

29. Li DG, Jin JM, Jiang ZZ. Pathological drunkenness: a case report. Yanbian Medical Journal. 1985;8(3):180-181. Chinese. 


\section{Publish your work in this journal}

Neuropsychiatric Disease and Treatment is an international, peerreviewed journal of clinical therapeutics and pharmacology focusing on concise rapid reporting of clinical or pre-clinical studies on a range of neuropsychiatric and neurological disorders. This journal is indexed on PubMed Central, the 'PsycINFO' database and CAS,

Submit your manuscript here: http://www.dovepress.com/neuropsychiatric-disease-and-treatment-journal and is the official journal of The International Neuropsychiatric Association (INA). The manuscript management system is completely online and includes a very quick and fair peer-review system, which is all easy to use. Visit http://www.dovepress.com/testimonials.php to read real quotes from published authors. 